\title{
Ambulatory Assessment in the Research on Aging: Contemporary and Future Applications
}

\author{
Annette Brose ${ }^{a, b} \quad$ Ulrich W. Ebner-Priemer ${ }^{c, d}$ \\ ${ }^{a}$ KU Leuven, Leuven, Belgium; ${ }^{b}$ Institute of Psychology, Humboldt University Berlin, Berlin, ${ }^{C}$ Karlsruhe Institute of \\ Technology, Karlsruhe, and ${ }^{\mathrm{d}}$ Central Institute of Mental Health, Mannheim, Germany
}

\section{Key Words}

Ambulatory assessment · Aging · Well-being paradox · Intervention - Emotion regulation - Tipping points

\begin{abstract}
Older adults have surprisingly high levels of well-being, which has been referred to as a paradox in the past. Improved emotion regulation has been suggested to underlie these high levels of well-being. Later life is also a period with enhanced exposure to critical life events, and this comes with risks. During such times, and towards the end of life, emotional well-being may and eventually does decline. We suggest that ambulatory assessment (AA) is ideally suited for the investigation of the above phenomena and for intervention purposes. More precisely, AA can be used to thoroughly examine within-person processes of emotion regulation, including the multiple levels on which emotions occur (physiology, experience, behavior, context, and nonverbal expressions). It thereby provides a basis for understanding competent emotion regulation, the well-being paradox, and emotionally critical periods. Such insights can be utilized to detect person-specific critical periods and for designing immediate person-specific interventions. Although this is still a vision, the benefits of such an approach seem invaluable.
\end{abstract}

The major part of this paper is organized around three general principles that we suggest to further tap the potential of AA in aging research, namely (1) identify within-subject processes and their relations to important life outcomes; (2) capitalize on the full scope of AA technology via multivariate assessments, and (3) combine real-time monitoring with real-time interventions.

(c) 2015 S. Karger AG, Basel

Ambulatory assessment (AA) is a methodology for assessing peoples' ongoing thoughts, feelings, behaviors, and biological processes during daily life experiences (www.ambulatory-assessment.org). Alternative labels for this methodology are ecological momentary assessment and experience sampling methodology. Its use in psychological research is rapidly growing due to its convincing advantages [1]: (a) findings from AA are ecologically valid because they are collected in the most ecologically valid situation possible, in people's everyday lives; (b) using real-time assessment self-reports is less prone to memory biases and other biases than using retrospective questionnaires; (c) repeated observations allow for the study of mechanisms and processes that underlie behavior, and (d) the data are rich in that contextual information can be

\section{KARGER 125}

(c) 2015 S. Karger AG, Base

$0304-324 \mathrm{X} / 15 / 0614-0372 \$ 39.50 / 0$

E-Mail karger@karger.com

www.karger.com/ger
Annette Brose

Institute of Psychology

Humboldt University Berlin, Rudower Chaussee 18

DE-12489 Berlin (Germany)

E-Mail annette.brose@hu-berlin.de 
gathered in addition to person-specific information. In multiple fields of research, new insights are gained by means of AA, including, for instance, research on emotions, health, developmental psychology and psychopathology [2].

The purpose of this paper is to demonstrate the potential of AA for the research on aging (for previous reviews on the use of AA in aging research, see Cain et al. [3] and Hoppmann and Riediger [4] $)^{1}$. We start with a brief overview of the contemporary use of AA in aging research, covering health-related processes, physical activity, cognitive functioning, and psychological features. Subsequently, we suggest that AA holds a key for understanding mechanisms that underlie resilience and well-being in later adulthood and that AA can be used to preserve wellbeing.

\section{Contemporary Use of AA in the Research on Aging}

\section{AA Research on Phenomena Prone to Age-Related Decline}

The AA of health-related processes in older adults in daily life comprises the assessment of physiological parameters such as cardiovascular activity (e.g., the continuous monitoring of blood pressure in daily life [5]). It is also used to monitor self-reported symptoms and symptom management [6]. Relatedly, AA allows for the examination of social interactions related to health, such as caregiving [7]. Finally, ambulatory technology is used to promote beneficial health behaviors (see below).

A second theme of aging research that is approached with AA is (physical) activities in daily life. Accelerometers can be used to measure sleep duration, active and sedentary behaviors, and physical activity energy expenditure [8], often with the aim of establishing relationships with health and well-being or in combination with feedback to reinforce physical activity [3].

AA studies on cognitive functioning in daily lives are still rare but nevertheless insightful. They have revealed that performance variability in older adults is related to affect, control beliefs, and physiological indicators of arousal $[9,10]$. For example, Riediger et al. [10] found that tense arousal and physiological activation were accompanied by impairments in cognitive performance in

\footnotetext{
1 Note that the following sections indicate recent developments; we primarily refer to the literature that was published after 2009.
}

Ambulatory Assessment in the Research on Aging middle and later adulthood. These findings indicate the importance of restraining oneself from hectic schedules in old age and from engaging in cognitively demanding activities, such as driving in times of stress [9].

\section{AA Research on Competencies and Potential Gains in Older Adulthood}

Themes of AA studies that are related to competencies in later adulthood are affective-motivational experiences, the management of challenges in daily life (e.g., daily stressors), and social contexts and interactions in old age. A thorough review on those topics is provided by Hoppmann and Riediger [4] and will thus be touched on very briefly here. Findings on affective experiences in daily life generally support the positive picture of emotional aging, which is certainly a domain of well-preserved functioning in later adulthood [11]. To give just two examples, older adults' emotional daily lives are more stable than those of younger adults, and older adults are more motivated and successful than younger adults to maintain positive states $[12,13]$. In summary, current AA research contributes to understanding age-related decline and competencies in later adulthood.

\section{The Potential of AA for Understanding and Maintaining Well-Being in Older Adulthood}

We propose three principles in the following sections to tap the full potential of AA in research on aging (table 1): (1) identify within-subject processes and their relations to important life outcomes; (2) capitalize on the full scope of AA technology via multivariate assessments, and (3) combine real-time monitoring with real-time interventions. The first two principles build on existing research and can be viewed as refinements. Our major emphasis will be on interventions, particularly on how and why AA is ideally suited to identify optimal times and individualized strategies for interventions with the ultimate goal of maintaining well-being in later adulthood.

Our focus on well-being is based on the following considerations. First, the question why older adults feel so well despite losses, referred to as the well-being paradox, continuous to draw researchers' attention [14]. One explanation is that emotion regulation competencies improve with age [11]. Surprisingly little is known, however, on age differences in actual processes of emotion regulation. We propose that AA is a promising tool to understand these processes and whether they underlie the wellbeing paradox. Second, much research on emotional 
Table 1. Overview on three principles for the understanding and maintenance of well-being in older adulthood by means of AA

\begin{tabular}{llll}
\hline & $\begin{array}{l}\text { Within-subject processes } \\
\text { and outcomes }\end{array}$ & Multivariate assessment & $\begin{array}{l}\text { Linking monitoring and } \\
\text { interventions }\end{array}$ \\
\hline Focus of this review & $\begin{array}{l}\text { Emotion regulation } \\
\text { and well-being }\end{array}$ & $\begin{array}{l}\text { Self-report, GPS, cardiovascular } \\
\text { and hormonal activity, voice } \\
\text { recordings, accelerometry }\end{array}$ & $\begin{array}{l}\text { Use of AA to detect critical points in } \\
\text { the emotion system's dynamics, } \\
\text { immediate intervention }\end{array}$ \\
$\begin{array}{l}\text { Exemplary research } \\
\text { question }\end{array}$ & $\begin{array}{l}\text { Are higher levels of well-being } \\
\text { the cause of (potentially) improved } \\
\text { emotion regulation in later } \\
\text { adulthood? }\end{array}$ & $\begin{array}{l}\text { Is the picture on (potentially) } \\
\text { improved well-being consistent } \\
\text { across different indicators? }\end{array}$ & $\begin{array}{l}\text { Can critical periods be detected and } \\
\text { do instructions help to stop a } \\
\text { downward spiral? }\end{array}$ \\
\hline
\end{tabular}

well-being in later adulthood has relied on self-report. We think that a validation of these findings is highly desirable and that the technological facilities of AA provide optimal means for a multivariate assessment of well-being. Third, not all older adults are blessed with high levels of well-being, and well-being declines at some point in life [15]. We propose that AA is a tool to detect critical periods in older adults' daily lives (i.e., times with enhanced psychological vulnerability) and to tailor person- and context-specific interventions.

\section{Linking Process and Outcome to Understand the Well-Being Paradox}

AA is ideally suited to test the yet rarely tested assumption that age-related improvements in emotion regulation are one reason for high levels of well-being in adulthood.

\section{The Emotion Regulation Process}

The essence of emotional and emotion regulation processes is that they evolve across time and that they occur in specific contexts. AA can target this essence and pinpoint competent emotion regulation, which refers to the appropriate tailoring of strategies to situations, resources, and global goal structures $[13,16,17]$. Potential age-related improvements in emotion regulation can be aptly addressed with AA because life as it is lived comes with exposure to different types of events, and with the use of multiple emotion regulation strategies that should be better tailored to situations with increasing age. Furthermore, the time course of emotional episodes entails information on emotion regulation competencies (i.e., the strength and pace of the reaction, the duration and profile of the episode, with moderate and circumscribed episodes being indicative of competent emotion regulation $[18,19])$. Data that are acquired with AA are ideally suit- ed to analyze the time courses of emotional episodes and to examine age group differences therein (see Carstensen et al. [12] and Wrzus et al. [20] for pioneering work in this regard). The same applies to the use of emotion regulation strategies. The competent use of strategies also manifests as specific patterns across time and should be examined accordingly. Relatedly, emotion regulation is competent if it reduces negative feelings. This proposition requires investigating interactions between strategy use and emotional episodes (e.g., whether affective reactivity is reduced in the presence of specific strategies [21,22]). Research on emotional aging uses AA and within-person data analytical approaches, but the time courses and contextual specificity of emotion regulation often remain to be examined.

\section{Linking Emotion Regulation with Outcomes}

Signs of improved emotion regulation in later adulthood as approachable with AA and, as described above (the situation-specific and effective use of emotion regulation strategies, moderate and circumscribed emotional reactions), are sometimes interpreted as evidence for the proposition that competent emotion regulation causes high levels of well-being in older adults and thus can explain the well-being paradox. This sounds plausible, but scrutinizing the current empirical evidence alone does not warrant this conclusion. That is, current AA studies on emotional aging that investigate emotional dynamics in different age groups often reveal age group differences, but they only infer relations between emotional dynamics and emotional outcomes. Following the recent argumentation by Isaacowitz and Blanchard-Fields [23] on whether cognition works in the service of well-being in later adulthood, we indicate the necessity to link emotion regulation and outcomes. An example for this idea is the study by Pitzer and Bergeman [24], who related affect de- 
synchrony (i.e., an inverse association between positive and negative affect that is indicative of regulatory competencies) after the loss of one's partner with grief (the outcome). AA is particularly well suited to investigate such topics; emotional processes can easily be linked with outcomes because the repeated assessment provides the data to analyze processes as well as to compute reliable means for indicating well-being. We thus suggest that AA studies directly test whether emotion regulation and well-being are related and whether this explains age-related differences in well-being. Finally, to establish evidence for mediation, both well-being and emotion regulation must be measured longitudinally and with measurement burst designs (i.e., longitudinal studies with intense measurements at each wave) [25]. These data can be used to determine whether affect regulation explains changes in well-being.

\section{Multivariate Assessments}

The potential of AA extends far beyond the assessment of subjective experiences. Capitalizing on the full scope of AA, including physiological and behavioral parameters, comes with at least two advantages in research on emotional aging. It provides a possibility to validate self-reports and it is an adequate representation of the complex nature of emotional episodes.

\section{The Validity of Self-Reports}

Studies that use AA have revealed relatively low variability and mean levels in older adults' self-reported affects [26]. One might conclude from this pattern that older adults rarely experience emotional episodes, or that the central outcome measure is not entirely informative about emotional processes. A recent review on how information processing varies as a function of the reference timeframe is illuminating in this regard. Conner and Barrett [27] argue that self-reports of concurrent experiences in AA studies are based on the 'experiencing self' (i.e., they are tied to sensory information). Instead, reports in trait questionnaires are more likely based on the 'believing self (i.e., they are based on long-term memory). It could be speculated whether older adults' reports of concurrent experiences in AA studies are based on the believing rather than the experiencing self. Such an answering schema would be efficient in view of age-related declines in interoception and limited cognitive resources. This possibility seems plausible in light of experimental findings in which individuals with low encoding skills evaluate their current state in accordance with personality traits; their reports seem to reflect their beliefs rather

Ambulatory Assessment in the Research on Aging than their actual feelings [28]. It would thus be advantageous to examine bodily responses during emotional episodes with continuous ambulant recording (e.g., cardiovascular activity, event-based collections of salivary cortisol). Smartphones can also be used to photograph concurrent facial expressions, and voice recordings can be informative about the changes in the tone of language. Combining these ways to measure emotions can reveal whether emotional episodes of older adults are indeed captured and not overlooked as a result of relying on selfreports.

Complex Representations of Emotional Responding

A multivariate approach to emotional responding is, of course, a more appropriate reflection of the nature of emotions. First steps have been made in this direction in aging research. For example, Wrzus et al. [29] combined phone-based AA with an ambulatory psychophysiological monitoring system for $24 \mathrm{~h}$. They showed increased psychological and cardiovascular responding to complex negative events with increasing age. Another example is the study by Nater et al. [30]: monitoring cortisol secretion and alpha-amylase output in older adults' daily lives was feasible in their study. This implies that hormonal changes can be investigated in ecologically valid contexts in that age group. Studies like these can reveal how emotional response components are differentially related to well-being. Thus, they can pinpoint components of emotional processes with most detrimental effects on physiological outcomes and thereby provide highly relevant insights for interventions.

\section{Intervening with $A A$ and the Prevention of Decline in} Well-Being

Despite generally high levels of well-being in later adulthood, there is considerable heterogeneity across individuals, including the incidence of depression and other mood disorders in older adulthood. Well-being is also challenged repeatedly throughout later life by the exposure to losses, and it almost inevitably declines at some point, during times of terminal decline [31]. The enhanced physiological vulnerability in later adulthood strengthens the relationship between psychological crises and physical health in this age group (e.g., enhanced effects of stress on health because of weaker immune systems). It thus seems essential to identify risk factors for well-being in later adulthood and to prevent maladjustment. Important risk factors in the old age are contextual challenges (e.g., the loss of a close other). The following sections describe (a) how AA is ideally suited to detect 
Table 2. Ambulatory monitoring and interventions

Assessment/monitoring: detection of critical periods WHEN to intervene?

Spotting thresholds and critical events in the existence of clear criteria

Example: change in caregiving responsibilities

Monitoring of processes, detection of changes in patterns of

covariation

(a) Theory-based detection of critical patterns

(b) Exploratory detection of change in a system

Examples: increased stress reactivity, slowing down of emotional processes

Self-monitoring of feedback

Example: spotting dysfunctional patterns of behavior, such as an increase in the discrepancy between actual and desired states

Assessment/intervention: providing support during critical periods

HOW to intervene?

Generic interventions

Example: prompts to engage in reappraisal when confronted with a stressor

Individualized interventions

(a) Insights from monitoring

(b) Assessment of personally relevant strategies

Examples: suggesting coping strategies that successfully reduced negative affect in the past/that subjectively ameliorate distress

Interactive interventions

(a) Interactions with practitioners in the health care system

(b) Automated involvement of close others

Example: automatic messages to practitioners or close others in critical moments, potentially including (automatic) responses

such challenges and periods that require interventions and (b) that AA is an effective means to exert immediate, individualized interventions. Table 2 provides an overview on these topics.

\section{The Identification of Critical Periods by Means of}

AA

Preventing declines in well-being requires the identification of critical periods. We suggest three approaches for the identification of such periods, namely the detection of (1) critical events or thresholds that indicate maladjustment, (2) changes in emotional and behavioral dynamics, and (3) self-monitoring through the provision of feedback.
Critical Events and Thresholds

Critical life events are highly relevant for the onset of maladjustment. For example, disability in the old age is followed by decreases in well-being [32], and the incidence of depression is increased in older adults after exposure to critical life events such as bereavement [33]. Other types of critical events are increases in caregiving responsibilities or relocation to nursing homes. Interventions could be prompted by the incidences of such events or even in advance of foreseeable events. One could also define thresholds in the affective domain that mark the need for an intervention (e.g., levels of well-being that are below 1 SD of a person's average for an extended period of time, or feelings of hopelessness on $50 \%$ of a series of observations). The latter criteria are arbitrary, which is due to a lack of criteria that exist, for example, in interventions that address health behaviors (e.g., smoking cessation).

\section{Changes in Emotional and Behavioral Patterns}

Critical periods could also be revealed by less obvious changes, such as an increase in reactivity to daily stressors. Detecting such changes requires intense measurements and sophisticated data analysis. The advantage of combining AA with interventions becomes obvious at this point; continuously analyzing reactivity to stressors can reveal time-related changes. Moreover, the relevance of combining insights on emotional processes (see the section on emotional processes) with interventions seems crucial in this context because the former provide the basis for making meaning of changes in emotional dynamics. For example, basic research on bereavement has revealed increased affect variability during the early phases of bereavement [34]. Affect variability seems to reflect not only instability in this context but also an adaptation process. An intervention that would merely aim to reduce variability would be contraindicated in this particular context. That is, knowledge on emotional processes is the basis for appropriate interventions.

The detection of 'tipping points' is another example of how a priori knowledge can help to reveal critical periods by means of AA. Tipping points refer to moments when a system loses resilience and may shift to an alternative state, such as a fragile, emotional state in depression [35]. For example, the slowing down of the emotional system (i.e., less change in emotions from occasion to occasion) seems to be indicative of the onset of depression, potentially because it indicates insensitivity to emotional cues. The latter is discussed as a predictor of depression. This slowing down can be detected only if emotions are continuously monitored across time. 
If a priori knowledge is not available on how a system functions in proximity to a crisis, AA may nevertheless be beneficial for the detection of critical periods. The continuous monitoring of multiple indicators of well-being by means of AA allows for the real-time analysis of multivariate patterns of covariation among the variables (e.g., the covariation of self-reported affect, physical activity, and cardiovascular activity). There are exploratory data analytical tools that can be used to analyze the concordance and synchronization in such time series data, including the detection of potentially critical changes in the time series (i.e., DeCon [36]). A data-driven detection of changes may indicate the need for an intervention. To give two examples, a synchronization of affective experiences (e.g., sadness, anger, and fear) may indicate a loss of the ability to emotionally differentiate between situations. A decoupling of affective experiences from leisure activities may indicate a loss in feeling pleasure in daily life. Both could be viewed as critical changes and, in principle, could elicit an intervention.

Self-Monitoring through the Provision of Feedback

Another way to detect critical periods with AA is the provision of feedback to individuals who are being monitored. Such an approach provides the opportunity for self-monitoring and for gaining insights into ones patterns of behavior. To illustrate this, Kramer et al. [37] provided depressed patients with information on positive affect in different situations. The result of this intervention was a stronger decrease in depressive symptoms in comparison to a control group. One interpretation of this finding is that self-monitoring provided insights into beneficial and nonbeneficial contexts, which were accompanied by changes in behavior. One could thus speculate whether self-monitoring reveals, for example, increasing discrepancies between actual and ideal states or increasingly dysfunctional attitudes. Once an individual becomes aware of such changes, she or he could prompt an electronic intervention or search for other types of support. Critical periods could be detected similarly if one focused on other psychophysiological parameters. One could (a) define a level of heart rate in stressful situations that indicates the need for interventions, (b) intervene in the detection of changes in heart rate variability, or (c) realize situation-specific discomfort if provided with information on cardiovascular activity. The important point is that critical periods are partly time-dependent and thus require continuous monitoring to be detected.
Intervening during Critical Periods by Means of AA

Ambulatory technologies are no longer used merely to monitor people. They are also used as tools for interventions, referred to as AA interventions (AAI) [1] or ecological momentary interventions [38]. They range from the provision of general informational support (e.g., reminders to engage in a specific behavior) to personalized interactive interventions. The latter examine current states of single individuals, analyze them in real time and give feedback if necessary and appropriate. Two examples might help to illustrate their potential. Ebner-Priemer et al. [8] developed an algorithm that assesses and analyzes physical activity in real time and poses questions according to the current physical state of a person. Such an algorithm could be used to advise older people to increase their physical activity not during their physician's visit but on a specific day on which they did not meet their preselected activity goals. It may also be used to positively reinforce older people during moments when they perform physical activity. Another example is the randomized clinical trial by Gustafson et al. [39], in which a GPSbased smartphone app successfully supported recovery from alcohol use disorder by triggering patients and offering support when they neared their favorite bars. The remainder of this section provides an overview on how AAI can be used to ameliorate or overcome emotionally critical periods. Our systematization was inspired by recent reviews of AAI in the domains of health and psychopathology $[38,40]$. From a technological point of view, the methods developed in health sciences can be easily adapted.

\section{Generic Content AAI}

The provision of information with generic content is the simplest form of AAI. Examples are prompts to use specific emotion regulation strategies that are known to be more beneficial than others or to engage in activities that are generally known to enhance well-being (e.g., taking rests during stressful times). Strategy selection should be based on insights from basic research. Such interventions lack specificity, of course, but they can be used easily for large scale interventions.

\section{Individually Tailored Automatic AAI}

Tailoring individualized interventions can take two forms. First, personally relevant strategies or goals for interventions can be assessed prior to AAI in addition to the assessment of preferred timeframes for ambulatory interventions (e.g., learning whether prompts should start at 7 or 10 a.m. and during what times or situations 
interventions seem particularly relevant). For example, an individual could specify resources that he or she wants to be reminded of in critical periods (i.e., activities that prevented negative states in the past). Second, AAI can be automatically tailored to individuals' needs through AA. For example, AA can monitor the specific locations that an individual visits (via GPS), physical activities in which an individual engages (via accelerometers), and significant others (via voice recordings/face detection algorithms). When this information is recorded along with affective experiences, contexts can be differentiated according to their effects on mood (e.g., within-person associations can be established between current affect and social contacts). Once such if-then contingencies are identified, specific advice can be given via mobile devices. For example, individuals could be automatically advised to search for significant others or specific places and reconsider the use of emotion regulation strategies in specific situations, depending on an algorithm that was developed during an assessment phase. Continued monitoring during interventions then also provides the opportunity to adapt the intervention itself in the sense of aptitude-treatment interactions [41].

\section{Automated Social Interactions}

AAI can also be used in an interactive manner. Practitioners in the health care system or predetermined close others could serve as interaction partners to whom people could turn via electronic devices to indicate the need for support. Social interaction partners could also be automatically prompted in predefined moments (e.g., when mood levels drop below a certain threshold). Feedback does not necessarily have to be personal but can remain automated. Such a procedure was successfully implemented by Spaniel et al. [42]. In their study, patients reported psychotic symptoms electronically, which elicited a psychiatrist's actions. An advantage of automated social interactions might be that people seek support earlier because disclosure is somewhat less personal.

The different types of AAI just outlined have one thing in common: they provide support in people's everyday lives [38]. The ecological validity of such interventions is thus high in the sense that strategies and skills can be applied to real-world problems. An additional benefit of AAI, if used in combination with AA, is the option to adapt intervention algorithms if a suggested behavior repeatedly fails to help.

\section{Conclusion}

In this review, we have suggested three principles to further tap the potential of AA in aging research: (1) identify within-subject processes and their relations to important life outcomes; (2) capitalize on the full scope of AA technology via multivariate assessments, and (3) combine real-time monitoring with real-time intervention. Pursuing these principles in the study of emotional aging shall reveal (1.1) which emotional processes underlie well-being in later adulthood; (2.1) which parameters (subjective and objective) to consider in the study of well-being, and (3.1) the nature of critical periods that may hallmark the beginning of maladjustment in old age and thereby times during which older adults may benefit from an intervention. Basic research on the first and second principles can be observed as a requirement for appropriate interventions, as is suggested in the third principle.

The use of $\mathrm{AA}$ in the research on aging also requires a cautionary note. In general, AA seems not to be greatly affected by reactivity [1], but empirical evidence on this issue is still limited [43]. Mood reports may be affected by their intense measurement, and compliance may change across a study's time span [44]. Investigations of these issues in the group of older adults are desirable. A second concern is the question whether older adults are able to handle AA technologies and how to address the potential damage or loss of the devices. Thorough instructions on how to use AA technologies are the basis for proper research, and to achieve the latter, it is advisable to provide a study reimbursement after the devices are returned. Compliance is a third issue that is debated in research on AA. The successful use of AA in severely ill patients (e.g., low dropout rates, reactions to large percentages of prompts in patients with borderline personality disorder or psychosis) [1] gives reason to be optimistic in this regard, and current studies with older adults support this conclusion. We are optimistic that an increasing use of AA technologies will show its added value and that the effects of the measurement procedure itself are largely outweighed by its advantages.

In conclusion, pursuing the three principles suggested in this viewpoint seems highly desirable to further validate the proposition that improved emotion regulation underlies high levels of well-being in later adulthood. Additionally, given the heterogeneity of emotional development in the old age that is often overlooked $[4,23]$, insights on competent emotion regulation can be utilized to design interventions for older adults with low levels of well-being. Finally, late life is a period with enhanced ex- 
posure to critical events, which comes with risks. It is highly desirable to detect the potential loss of resilience as early as possible and to intervene such that the needs of individuals are met. Although this is still a vision, it seems possible that these goals are met through the use of AA.

\section{Acknowledgment}

This research was supported by a grant of the German Research Foundation (DFG, BR 3782/2-1) awarded to Annette Brose.

\section{References}

$>1$ Trull TJ, Ebner-Priemer U: Ambulatory assessment. Annu Rev Clin Psychol 2013;9: 151-176.

2 Mehl MR, Conner TS: Handbook of Research Methods for Studying Daily Life. New York, Guilford, 2012.

3 Cain AE, Depp CA, Jeste DV: Ecological momentary assessment in aging research: a critical review. J Psychiatr Res 2009;43:987-996.

$\checkmark 4$ Hoppmann CA, Riediger M: Ambulatory assessment in lifespan psychology: an overview of current status and new trends. Eur Psychol 2009;14:98-108.

$\checkmark 5$ Hodgkinson JA, Sheppard JP, Heneghan C, Martin U, Mant J, Roberts N, et al: Accuracy of ambulatory blood pressure monitors: a systematic review of validation studies. J Hypertens 2013;31:239-250.

-6 Quandt SA, Grzywacz JG, Neiberg RH, Lang W, Altizer K, Bell RA, Arcury TA: Daily symptom management practices for arthritis used by older adults. J Aging Health 2012;24: 598-615.

$>7$ Koerner SS, Shirai Y, Kenyon DB: Sociocontextual circumstances in daily stress reactivity among caregivers for elder relatives. J Gerontol B Psychol Sci Soc Sci 2010;65B:P561P572.

8 Ebner-Priemer U, Koudela S, Mutz G, Kanning $\mathrm{M}$ : Interactive multimodal ambulatory monitoring to investigate the association between physical activity and mood. Front Psychology 2013;3:596.

$>9$ Neupert SD, Patterson TR, Davis AA, Allaire JC: Age differences in daily predictors of forgetting to take medication: the importance of context and cognition. Exp Aging Res 2011; 37:435-448.

$>10$ Riediger M, Wrzus C, Klipker K, Muller V, Schmiedek F, Wagner GG: Outside of the laboratory: associations of working-memory performance with psychological and physiological arousal vary with age. Psychol Aging 2014;29:103-114.

$>11$ Scheibe S, Carstensen LL: Emotional aging: Recent findings and future trends. J Gerontol B Psychol Sci Soc Sci 2010;65B:P135-P144.

12 Carstensen LL, Pasupathi M, Mayr U, Nesselroade JR: Emotional experience in everyday life across the adult life span. J Pers Soc Psychol 2000;79:644-655.
3 Riediger M, Schmiedek F, Wagner GG, Lindenberger U: Seeking pleasure and seeking pain: age-related differences in pro- and contra-hedonic motivation from adolescence to old age. Psychol Sci 2009;20:1529-1535.

14 Kunzmann U, Little TD, Smith J: Is age-related stability of subjective well-being a paradox? Cross-sectional and longitudinal evidence from the Berlin Aging Study. Psychol Aging 2000;15:511-526.

15 Gerstorf D, Ram N, Schupp J, Lindenberger $\mathrm{U}$, Wagner GG: Late-life decline in well-being: replicating and extending evidence from the Berlin Aging Study using national surveys from Germany, the UK, and the US. Gerontologist 2009;49:53.

16 Blanchard-Fields F, Mienaltowski A, Seay RB: Age differences in everyday problem-solving effectiveness: older adults select more effective strategies for interpersonal problems. J Gerontol B Psychol Sci Soc Sci 2007;62B:P61P64.

17 Urry HL, Gross JJ: Emotion regulation in older age. Curr Dir Psychol Sci 2010;19:352-357.

18 Koole S: The psychology of emotion regulation: an integrative review. Cogn Emot 2009; 23:4-41.

$>19$ Verduyn P, Van Mechelen I, Tuerlinckx F, Meers K, Van Coillie H: Intensity profiles of emotional experience over time. Cogn Emot 2009;23:1427-1443.

20 Wrzus C, Müller V, Lindenberger U, Wagner GG, Riediger M: Affect dynamics across the lifespan: with age, heart rate reacts less strongly, but recovers more slowly from emotional strain. Psychol Aging, in press.

21 Brose A, Schmiedek F, Lövdén M, Lindenberger U: Normal aging dampens the link between intrusive thoughts and negative affect in reaction to daily stressors. Psychol Aging 2011;26:488-502.

22 Kuppens P, Oravecz Z, Tuerlinckx F: Feelings change: accounting for individual differences in the temporal dynamics of affect. J Pers Soc Psychol 2010;99:1042-1060.

23 Isaacowitz DM, Blanchard-Fields F: Linking process and outcome in the study of emotion and aging. Perspect Psychol Sci 2012;7:3-17.

24 Pitzer LM, Bergeman CS: Synchrony in affect among stressed adults: the Notre Dame Widowhood Study. J Gerontol B Psychol Sc 2014; 69:P29-P39.
25 Ram N, Coccia M, Conroy D, Lorek A, Orland B, Pincus A, et al: Behavioral landscapes and change in behavioral landscapes: a multiple time-scale density distribution approach. Res Hum Dev 2013;10:88-110.

26 Röcke C, Brose A: Intraindividual variability and stability of affect and well-being. Shortterm and long-term change and stabilization processes. GeroPsych 2013;26:185-199.

27 Conner TS, Barrett LF: Trends in ambulatory self-report: the role of momentary experience in psychosomatic medicine. Psychosom Med 2012;74:327-337.

28 Robinson MD, Clore GL: Traits, states, and encoding speed: support for a top-down view of neuroticism/state relations. J Pers 2007;75: 95-120.

29 Wrzus C, Muller V, Wagner GG, Lindenberger U, Riediger M: Affective and cardiovascular responding to unpleasant events from adolescence to old age: complexity of events matters. Dev Psychol 2013;49:384-397.

30 Nater UM, Hoppmann CA, Scott SB: Diurnal profiles of salivary cortisol and alpha-amylase change across the adult lifespan: evidence from repeated daily life assessments. Psychoneuroendocrinology 2013;38:3167-3171.

31 Gerstorf D, Ram N: Inquiry into terminal decline: five objectives for future study. Gerontologist 2013;53:727-737.

32 Lucas RE: Long-term disability is associated with lasting changes in subjective well-being: evidence from two nationally representative longitudinal studies. J Pers Soc Psychol 2007; 94:717-730.

-33 Kraaij V, Arensman E, Spinhoven P: Negative life events and depression in elderly persons: A meta-analysis. J Gerontol B Psychol Sci Soc Sci 2002;57B:87-94.

34 Bisconti TL, Bergeman CS, Boker SM: Emotional well-being in recently bereaved widows: A dynamical systems approach. J Gerontol B Psychol Sci Soc Sci 2004;59B:P158P167.

35 van de Leemput IA, Wichers M, Cramer AOJ, Borsboom D, Tuerlinckx F, Kuppens P, et al: Critical slowing down as early warning for the onset and termination of depression. Proc Natl Acad Sci USA 2014;111:87-92.

36 Bulteel K, Ceulemans E, Thompson RJ, Waugh CE, Gotlib IH, Tuerlinckx F, et al: DeCon: a tool to detect emotional concordance in multivariate time series data of emotional responding. Biol Psychol 2014;98:29-42. 
-37 Kramer I, Simons CJ, Hartmann JA, MenneLothmann C, Viechtbauer W, Peeters F, Schruers K, van Bemmel AL, Myin-Germeys I, Delespaul P, van Os J, Wichers M: A therapeutic application of the experience sampling method in the treatment of depression: a randomized controlled trial. World Psychiatry 2014; 13:68-77.

38 Heron KE, Smyth JM: Ecological momentary interventions: incorporating mobile technology into psychosocial and health behaviour treatments. Br J Health Psychol 2010;15:1-39.
9 Gustafson DH, McTavish FM, Chih MY, Atwood AK, Johnson RA, Boyle MG, et al: A smartphone application to support recovery from alcoholism: a randomized clinical trial. JAMA Psychiatry 2014;71:566-572.

40 Clough BA, Casey LM: Technological adjuncts to enhance current psychotherapy practices: a review. Clin Psychol Rev 2011;31: 279-292.

41 Cronbach L, Snow R: Aptitudes and Instructional Methods: A Handbook for Research on Interactions. New York, Irvington, 1977.
2 Spaniel F, Vohlídka P, Hrdlicka J, Kozený J, Novák T, Motlová L, Cermák J, Bednarík J, Novák D, Höschl C: ITAREPS: information technology aided relapse prevention programme in schizophrenia. Schizophr Res 2008;98:312-317.

43 Barta WD, Tennen H, Litt M: Reactivity in diary studies; in Mehl MR, Conner TS (eds): Handbook of Research Methods for Studying Daily Life. New York, Guilford, 2012.

44 Conner TS, Reid KA: Effects of intensive mobile happiness reporting in daily life. Soc Psychol Personal Sci 2012;3:315-323. 